\title{
A review of using waste and virgin polymer in pavement
}

\author{
Zahra Niloofar Kalantar, Mohamed Rehan Karim, Abdelaziz Mahrez
}

Introduction

The use of plastic bottles throughout the world is on the increase

[1-4]. Both the creation and the recycling procedures of

plastic bottles are detrimental to the environment [5-9]. Plastics

do not decompose naturally and so alternative methods need to

be implemented, in order to recycle the plastic material.

The performance of road surfaces can be improved by modifying

bitumen [10-18]. There are numerous modifiers that can be

used to improve the properties of road surfaces, but most of these

are virgin materials. Virgin materials are difficult to find and are uneconomical when used as a modifier [19].

Therefore using waste plastic bottles as modifier in road surfaces can potentially help reduce

material wastage and improve the performance of road surfaces

at the same time [20-24].

History of using polymer in asphalt

Synthetic and natural polymers have been used in asphalt as a

modifier as early as 1843. In the 1930s the project was underway

in Europe and North America began to use rubber latex in 1950s.

Europe was using modified asphalts ahead of the United States

which were limited to use PMA because of its high expenses in

the late 1970s $[25,26]$. In the mid-1980s, US began to use new

developed polymers and European technologies. Currently in

Australia, polymer modified binders is included in the guides and

specifications of National Asphalt Specification [26].

In the survey of state departments of transportation in 1997, 47

states of US reported that in the near future they would be using

modified asphalts and 35 states reported that they would need bigger

portions. Several investigations all around the world have researched

and evaluated benefits of modifying polymers on the 
performance of pavement, and developing the specifications and tests for binders are still continuing [26].

Over the last decade, USA is the country where most of the research is done, followed by China, Canada and some European countries. Among the companies that have been filing patents on PMA over the last decade, Marathon Ashland Petroleum LLC is the leading one. The Goodyear Tire and Rubber Company, Fina Technology, Polyphalt LLC, BASF Corporation and Ergon Incorporated are also reported. There have been significant movements in the marketing area. The interest for PMA technology has been increasing, and so the number of companies which commercialize it, and their earnings [27].

The United States, China, France and Italy are leaders in polymer modified asphalt (PMA) research and development activities, even though considerable work has also been done in Japan, Germany, Russia, Great Britain, and Canada [27].

\section{The benefits of using polymer in asphalt}

Bitumen is one of the viscoelastic materials and the only deformable element of pavement and has a very important role in pavement performance [27]. Bitumen has a good adhesion and cohesion with aggregates therefore it has been used for roofing and paving purposes [27-32].

One of the most important properties of the bituminous mixture is its stability. The optimum stability is the one that can handle the traffic requirements sufficiently. If the stability is lower than the traffic demand, it will cause shoving and flow of the road surface [33]. To prevent a pavement from rutting the flow should be low. Flow can be considered as a property that reduces the stability $[34]$.

In hot climates rutting and in cold climates cracking are related to the sensitivity of the asphalt pavement to the temperature variation and the traffic load [35]. If the tire pressure, volume of heavy vehicles and traffic increases, a higher performance pavement will be needed which requires a bitumen with low susceptibility to temperature changes and has high adhesion to aggregates 
Some improvements in asphalt properties have been gained by selecting the proper starting crude, or tailoring the refinery processes used to make asphalt. Unfortunately, there are only a few crudes that can produce very good asphalts, and only a limited number of actions that can be taken to control the refining process to make improved asphalts [27]. The next step taken by the industry was to modify the asphalt. Air blowing makes asphalt harder [36-38]. Fluxing agents or diluent oils are sometimes used to soften the asphalt. Another method that can significantly improve asphalt quality is the addition of polymers [27]. TOSC0 (the 0il Shale Company), was the first company that used polyphosphoric acid (PPA) as a modifier in asphalt without air blowing. The other polymers that have been used together with PPA were, EVA [39, 40], GMA $[41,40]$, SIS, PE $[42,43]$, SBS $[44-50]$, APP $[51,52]$, ABS $[53,54]$, amongst several others. Modifying the asphalt with synthetic and natural polymers can improve the performance of roads [32] and blending is the most common method of modifying [31].

A significant number of researches on PMA (polymer modified asphalt) mixture have been conducted for the past two decades. The addition of polymers to asphalt for the purpose of enhancing its properties over a range of different temperatures in paving applications was contemplated a long time ago [55]. Polymers can significantly improve the asphalt pavements performance at low, intermediate and high temperatures. They can increase the resistance of mixture to permanent deformation, thermal fracture and fatigue cracking at low temperature, decrease plastic flow and increase shear modulus at high temperature $[35,31]$. The researchers found that by modifying bitumen with even small amounts of polymers, the road pavement life span may be increased [56].

Improvement in engineering properties including thermal cracking, stripping, rutting resistance, temperature susceptibility and fatigue damage, have led polymer modified binders to be a substitute for asphalt in paving and maintenance application, such as cold mix, cold and hot crack filling, slurry seal, patching, hot mix, chip seals and recycling. They also can be used to cut down the life cycle costs $[27]$.

\section{The use of waste polymer instead of virgin polymer}

It has been known for some time that virgin polymers can improve the modified bitumen' s properties, however, there are some 
concerns of replacing virgin materials with recycled polymers [31].

Since polymers are rather expensive, the amount of polymer used to improve the road pavement must be small. Recycled polymers have been found to show similar results in improving the road performance as compared to virgin polymers. From economic and environmental point of view using the waste polymer as a modifier is beneficial because using waste polymer as a modifier may help to improve the performance of pavement and quality of the roads and also to solve the waste disposal problem [31]. Many polymers have been used as binder modifiers, and they can be classified into five groups. Table 1 presents a summary of these polymers and their advantages and disadvantages as asphalt modifiers $[27]$

The polymers used to modify bitumen can be divided in two classes, elastomers and plastomers. Plastomers include polyethylene and various compounds based on polyethylene and ethylene vinyl acetate [57-60]. At normal temperature, these polymers can increase the stiffness of bitumen and provide a mix with high viscosity. Depending on the polymer, high shear mixing may be needed [57].

Full text available at :

http://www.sciencedirect.com/science/article/pii/S0950061812000323 